\title{
Ground-state properties of rutile: electron-correlation effects
}

\author{
Krzysztof Rościszewski, Klaus Doll, Beate Paulus, Peter Fulde \\ Max Planck Institut für Physik komplexer Systeme, Nöthnitzerstr. 38, D-01187 Dresden, Germany \\ Hermann Stoll \\ Institut für Theoretische Chemie, Universität Stuttgart, D-70550 Stuttgart, Germany
}

\begin{abstract}
Electron-correlation effects on cohesive energy, lattice constant and bulk compressibility of rutile are calculated using an ab-initio scheme. A competition between the two groups of partially covalent Ti-O bonds is the reason that the correlation energy does not change linearly with deviations from the equilibrium geometry, but is dominated by quadratic terms instead. As a consequence, the HartreeFock lattice constants are close to the experimental ones, while the compressibility is strongly renormalized by electronic correlations.
\end{abstract}

\section{INTRODUCTION}

Although transition-metal oxides are one of the most interesting classes of solids, relatively little theoretical work aiming at a microscopic understanding of electroncorrelation effects in these systems is available so far. This is not surprising: high numerical effort is already required here for accurately describing ground-state properties at the Hartree-Fock (HF) independent-particle level. Receptly, results on $\mathrm{NiO}^{2} \mathrm{O}$ and on the rutile $\mathrm{TiO}_{2}$ crystall $\mathrm{B}$ were published. Correlation effects in $\mathrm{TiO}_{2}$ have beep studied only implicitly, at the densityfunctional level|l 6 .

Rutile is one of the experimentally found modifications of $\mathrm{TiO}_{2}$. The investigation of the relative stability compared to other phases such as anatase, brookite or $\mathrm{TiO}_{2}(\mathrm{~B})$ is another interesting subject $\mathrm{d}$. Rutile is well studied by experimentalists; the structure was precisely determined by using X-ray and neutron diffraction (for a discussion of the experimental results see Ref. 6 and references therein). The experimental lattice constant is close to values obtained in ab-initio self-consistent field (SCF) calculationst, whereas the difference between SCF results and experiment for the compressibility is large. This contradiction makes the task of studying electron correlations in the rutile crystal interesting.

From the methodological point of view the rutile crystal is a next logical step in the application of a correlation treatment within the so-called "scheme of local increments" cessfully tested for covalently bonded solids like diamond, graphite and many typical semiconductors 9 . For purely ionic crystals the scheme works also we tsegreferences for $\mathrm{MgO}, \mathrm{CaO}, \mathrm{NiO}$ and alkali halides14.15, 2.16 ). II-VI semiconductors have been investigated as an example of partly ionic, partly covalent crystal 17 . With respect to this, rutile is another excellent object for testing.

In the present short contribution we report on ab-initio correlation calculations for the cohesive energy, lattice constant and compressibility of rutile. The paper is organized as follows. In the second chapter we outline the 
computational method and describe our results. We also discuss individual correlation-energy increments and address the question of their transferability when approximating the infinite crystal by embedded clusters. A discussion and a short summary are presented in the last chapter.

\section{COMPUTATIONAL METHOD}

The rutile structure is tetragonal (nonsymmorphic space group $P 4_{2} / \mathrm{mnm}$ ) with two titanium atoms and four oxygens per primitive unit cell (see Fig. 11). Ti atoms are located at positions $(0,0,0)$ and $(1 / 2,1 / 2,1 / 2)$ and oxygens at positions $( \pm x, \pm x, 0)$ and $(1 / 2 \pm x, 1 / 2 \mp x, 0)$ The lattice constants extrapolated to zero temperature 18 are $a=4.592 \AA$ and $c=2.958 \AA$, the dimensionless coordinate $x=0.3048$. The experimental value for $\theta$ is $98.8^{\circ}$. The bonding and charge distribution in the rutile crystal have been discussed in Ref. 5. The SCF Mulliken population analysis 3 shows that the excess negative charge on oxygen is about $-1.4 e$. On $\mathrm{Ti}$, in addition to the closed (but easily deformable) argon-like core the population analysis gives 1.2 valence electrons in the $d$ shell, whereas the $4 s$ shell is not occupied 3 , 2 .

After this preliminary discussion, we briefly review our theoretical approach for including electron correlation on top of a crystal SCF calculation. The main idea is to expand the total correlation energy, $E_{c o r r}$, of the crystal in terms of local correlation-energy increments. Details have been described in previous papers 811.14. Essentially, we use a Bethe-Goldstone-like hierarchy of the type

$$
\begin{array}{r}
E_{\text {corr }}=\sum_{A} \varepsilon(A)+\frac{1}{2} \sum_{A, B} \Delta \varepsilon(A B)+ \\
\frac{1}{3 !} \sum_{A, B, C} \Delta \varepsilon(A B C)+\ldots
\end{array}
$$

where $A, B, C, \ldots$ denote groups of occupied localized orbitals on atoms $A, B, C, \ldots$, respectively (we use the same symbol for an atom and for the group of the localized orbitals on $A$ ). The quantity $\varepsilon(A)$ (a one-body increment) denotes the (local) correlation energy of the crystal in which only the orbitals of group $A$ are correlated. The two-body increment $\Delta \varepsilon(A B)$ is defined as the nonadditive part of correlations arising from simultaneously correlating the groups $A$ and $B$ :

$$
\Delta \varepsilon(A B)=\varepsilon(A B)-\varepsilon(A)-\varepsilon(B)
$$

Similarly three-body increments can be introduced as follows:

$$
\begin{array}{r}
\Delta \varepsilon(A B C)=\varepsilon(A B C)-\Delta \varepsilon(A B)- \\
\Delta \varepsilon(A C)-\Delta \varepsilon(B C)-\varepsilon(A)-\varepsilon(B)-\varepsilon(C)
\end{array}
$$

Even if one should want to treat these formulae as purely phenomenological ones, still the expansion (if carried out to infinity) is exact which is clear upon inspection. (For a more sophisticated derivation, cf. Ref. 
19.) For practical applications, however, some approximations are inevitable. The first approximation consists in truncating the infinite series. The data for various systems $13.17 .24,16$ show that the expansion is quickly convergent. The three-body increments were found to be almost negligible (for the bulk of practical applications). Moreover, two-body increments were found to decay rapidly for larger distances. The second (more serious) approximation for the incremental expansion consists of replacing the infinite crystal with finite embedded clusters for the purpose of determining individual increments. This approximation uses the local nature of electron correlatione0 and was found to work well. Note that we restrict the local cluster treatment to correlation effects only - SCF interactions are long-range, and a calculation involving the whole (infinite) lattice is mandatory here this is possible with the CRYSTAL program package21.22.

Applying the combined scheme just described to $\mathrm{TiO}_{2}$, one obtains the total energy functional

$$
E_{t o t}(a, c, x)=E_{s c f}(a, c, x)+E_{\text {corr }}(a, c, x)
$$

as a function of the lattice parameters $a, c, x$ or $r_{1}, r_{2}, \Theta$ (in internal coordinates). The crystal compressibility $B$, at zero temperature, is defined as

$$
B=V \frac{\partial^{2} E_{0}(V)}{\partial^{2} V}
$$

with $V$ denoting the volume of the unit cell and $E_{0}(V)$ the conditional minimum of $E_{\text {tot }}(a, c, x)$ for fixed constant volume. Using $V=a^{2} c$ and expressing $E_{t o t}\left(a, V / a^{2}, x\right)$ as a function of two independent variables $a, x$, the compressibility $B$ can be obtained. This method is usually not applied to noncubic crystals, but instead the minimum position at $a_{0}, c_{0}, x_{0}$ is approached by a conjugated gradient technique. This reduces the number of data points, and the compressibility can only be obtained then by applying an empirical ansatz such as the Murnaghan equation of state (cf. e.g. Ref. (4). Another possibility is to define an artificial isotropic compressibility $B_{I S O}$ where one (incorrectly) assumes that under compression $x$ remains constant and $a$ and $c$ scale isotropically.

\section{A. Basis sets}

SCF calculations: For the titanium atom, a relativistic energy-consistent 12-valence-electrop pseudopotential23, together with a 411/411/41 basis set 4 was used. Fpr oxygen we chose the basis set given by Causà et al24. To calculate the energy of the free atoms, diffuse functions cannot be omitted (let us reming that they must be omitted in CRYSTAL calculations2122). Thus we appropriately supplemented the basis sets for this purpose 2 . Our SCF cohesive energy is smaller compared to that of Ref. A probably just because of the lack of diffuse functions 
for the free Ti atom in Ref. 1 . Of course, this omission affected only the cohesive energy.

Correlation calculations: For oxygen we used Dunning's correlation-consistent augmented valence triple zeta [ $5 s 4 p 3 d 2 f]$ basis set26. For Ti, our starting point was a $[6 s 5 p 3 d]$ basis set optimized for the pseudopotential of Ref. 23. We decontracted one $p$ and one $d$ function, ending up with a $[6 s 6 p 4 d]$ set which was supplemented with $2 f 1 g$ polarization exponents optimized in CCSD calculations for the free ground-state atom ( $f$ exponents are $2.45,0.766$, and the $g$-exponent is 2.132).

\section{B. SCF calculations}

With the CRYSTAL95 program package21.22, SCF energies for 100 different sets of randomly chosen $a, c, x$ values were calculated. A region around the minimum was covered more densely. The computational results were fitted by a 3 -variable polynomial of third order plus a few quartic terms which turned out to be significant. (Typically about twenty constants to fit.)

Some final results of SCF calculations are collected in Table I. We recover at the SCF level about $57 \%(834$ $\mathrm{mH}$ ) of the experimental cohesive energy. The lattice constant is already close to experiment, but the bulk modulus turns out to be substantially too high. These results indicate the importance of taking into account electron correlation.

\section{Correlation calculations}

For the correlation calculations, we applied the coupled-cluster approach with single and double excitations (CCSD) 27 and included perturbatively triples $(\operatorname{CCSD}(\mathrm{T}))^{28}$ as implemented in the program package MOLPRO29 30. Within the incremental scheme, we studied one-, two- and three-atom clusters. (For the threeatom clusters, we performed CCSD calculations only.) The clusters were embedded in a large slab of Madelung point charges $(7 \times 7 \times 9$ unit cells, charges 4 and -2 , respectively), similarly as described in Refs. $25,14,16$. An exception are the $\mathrm{Ti}$ ions nearest to the cluster atoms: in this case, $\mathrm{Ti}^{4+}$ pseudopotentials 3 were used instead of the bare point charges, in order to simulate the Pauli repulsion on the $\mathrm{O}^{2-}$ electrons of the inner cluster. The not fully ionic character of the system turns out to be the basic difficulty. When doing computations on finite clusters, only an integer electron number is allowed and the atomic populations obtained from the CRYSTAL calculation cannot easily be reproduced as is the case for perfectly ionic systems. We are forced to assign to the clusters considered the same total electron charges as in hypothetical purely ionic $\mathrm{Ti}^{4+}, \mathrm{O}^{2-}$ rutile. Of course, this is no issue in large clusters, but may be critical for 
small ones. In order to control the quality of this approximation, we compare the results for the individual increments taken from clusters with one, two, and three explicitly described ions (see Table II).

The bulk of our correlation-energy calculations (whose results appear in Table I) was done for embedded clusters with one and two explicitly treated atoms. The maximum distance between the atoms within these clusters was up to 8 atomic units. These clusters (altogether 13 in number) provide us with the most significant one-body and two-body contributions to $E_{c o r r}$. For 5 different geometries, all these increments were calculated. For 15 other geometries, the 8 most important increments were explicitly calculated whereas the remaining 5 least important (contributions below $1 \mathrm{mH}$ ) were obtained by interpolation. To estimate the importance of three-body contributions, we also calculated the biggest three-body $O-T i-O$ increments for one geometry, and they were found to be of the order of $1 \mathrm{mH}$.

A very important question is the transferability of these increments from one type of cluster to the other (see Table II). In every case, our increments refer to localized orbital groups which can be formally attributed to $\mathrm{O}^{2-}$ and $\mathrm{Ti}^{4+}$ ions, respectively, but due to partially covalent bonding in $\mathrm{TiO}_{2}$, especially the former orbital group becomes more delocalized when going to larger clusters. Specifically, we see that the oxygen correlation energy increases in magnitude when taken from a cluster with three explicitly described ions (one titanium ion, two oxygen ions, to be consistent with the formula unit) instead of out of a cluster where only the oxygen ion has basis functions. We explain this with a better description of the diffuse tail of the oxygen charge, as the charge is allowed to flow to the titanium ion. The more diffuse ion has lower-lying excitations which explains the change of the correlation energy. In the same way, we can compare the titanium correlation energy of a cluster where only one titanium ion has basis functions to that of a cluster with three explicitly described ions. We find that the titanium correlation energy is reduced in magnitude. This is due to the oxygen charge which has flown to the titanium ion and has led to a $d$ occupancy. Excitations of the $3 s$ and $3 p$ electrons into $d$ orbitals are now (partially) forbidden, i.e., we have an exclusion effect. In total, both effects almost cancel for the one-body increments $(|\Delta \varepsilon(O)|$ increases by $5 \mathrm{mH}$, to be multiplied with a weight factor of $4,(|\Delta \varepsilon(T i)|$ decreases by $11 \mathrm{mH}$, to be multiplied with 2). For the two-body increments, a partial cancellation with increasing cluster size takes place, too: $\mathrm{O}-\mathrm{O}$ increments are enhanced by $\sim 4 \mathrm{mH}$ when bridging $\mathrm{Ti}$ atoms are taken into account, while Ti-O increments are reduced in magnitude by $\sim 3 \mathrm{mH}$ in the $\mathrm{TiO}_{2}$ unit 32 .

As a whole, we find that the transferability is reasonable but rather poor in comparison to purely ionic crystals where cluster charges are strictly confined (see Table II). This is not unexpected as the perfect-ion approximation is broken in a different way for each of the clusters studied. To overcome this transferability problem, the 
only way would be to further improve the cluster surroundings by using more than three explicitly described ions with high quality basis set (ideally in multiples of the formula unit), which is presently not possible because of the steeply increasing computational effort.

The correlation energies obtained this way were fitted for 20 geometries by a second-order polynomial (ten parameters), analogously as previously described for SCF energies. Adding the two resulting formulae, we obtained $E_{t o t}(a, c, x)$ so that we could perform the calculation of physical constants which include correlation effects (see Table I).

\section{DISCUSSION AND SUMMARY}

As SCF and SCF +CCSD(T) lattice constants are not very different and both rather close to the experimental values we conclude that electron correlations do not influence the crystal geometry in a major way. The technical explanation is provided by studying the coefficients of the analytic (fitted) formula of $E_{\text {corr }}\left(r_{1}, r_{2}, \theta\right)$, in atomic units, at the $\operatorname{CCSD}(\mathrm{T})$ level:

$$
\begin{array}{r}
E_{\text {corr }}\left(r_{1}, r_{2}, \theta\right) \approx-2.1241-0.0121 \Delta r_{1} \\
-0.0067 \Delta r_{2}-0.0322 \Delta \theta \\
+0.2570\left(\Delta r_{1}\right)^{2}-1.5730\left(\Delta r_{2}\right)^{2}-1.2490(\Delta \theta)^{2} \\
-3.0459 \Delta r_{1} \Delta r_{2}+1.3580 \Delta r_{1} \Delta \theta-0.0875 \Delta r_{2} \Delta \theta
\end{array}
$$

The normalized dimensionless expansion variables are defined as $\Delta r_{1}=\left(r_{1}-r_{10}\right) / r_{1 \exp }$ where $r_{10}$ is the SCF equilibrium value of the internal variable $r_{1}$ and $r_{1 \exp }$ is the corresponding experimental value. Analogous expressions hold for $\Delta r_{2}$ and for $\Delta \theta$. The coefficients of the linear terms are much smaller than the coefficients of the quadratic terms. This explains why the compressibility is strongly renormalized by correlations as it depends primarily on the coefficients of the quadratic term.

To understand this situation it is necessary to study how individual increments change when the lattice constants change. To gain a qualitative understanding it is enough to study the biggest contributions: the one-body increment for oxygen and the two-body nn Ti-O increments. (Note that the one-body increment for Ti can be considered as constant, to a good approximation). In the following we will describe several competing mechanisms which cause linear changes of the bulk correlation energy to be small near the SCF minimum.

Let us start with oxygen (one-body increment for oxygen). Its change as a function of the lattice constant is in part of intraatomic origin and in part of electrostatic origin. First let us consider the intratomic part. As found and explained in earlier work 14. 15.2, excitations cost less energy when the lattice constant (and the volume of the quantum well enclosing the oxygen ion) increases; in that case, the magnitude of the correlation energy increases, 
too. There is an opposite trend connected with the permanent electric field $\vec{E}$ at the oxygen site. As the lattice expands, the Madelung field decreases, and the influence of correlation on the static polarization of the oxygen ions, $\frac{1}{2}\left(\alpha_{S C F}-\alpha_{c o r r}\right) \vec{E}^{2}$ (with polarizabilities $\alpha_{S C F}$ and $\alpha_{\text {corr }}$ at SCF and correlated levels, respectively), decreases in parallel. (Note that due to symmetry there is no static electric field at the site of the Ti ions). A competition between the two effects just described apparently leads to a relatively small change in the total oxygen correlation energy as a function of the lattice constant.

Thus, the most important source of variability are twobody increments. For the two-body nn Ti-O increments, we find two competing effects again. To fix the attention let us provide two greatly simplified formulae (atomic units):

$$
\begin{aligned}
\Delta \varepsilon_{\text {api }} & \approx-0.027+0.083 \Delta r_{1}-0.039 \Delta r_{2}-0.036 \Delta \theta \ldots \\
\Delta \varepsilon_{\text {equa }} & \approx-0.026-0.046 \Delta r_{1}+0.018 \Delta r_{2}+0.040 \Delta \theta \cdots
\end{aligned}
$$

where $\Delta \varepsilon_{a p i}$ and $\Delta \varepsilon_{\text {equa }}$ are two-body nn Ti-O increments for apical and equatorial oxygen ions, respectively. The large quadratic terms in the above formulae were not shown as they are irrelevant to the following arguments. Let us remember that the multiplying weight factors (per unit cell) for $\Delta \varepsilon_{a p i}$ and $\Delta \varepsilon_{\text {equa }}$ are 4 and 8 , respectively. Due to the opposite signs and due to the fact that the different linear term amplitudes are roughly in 1:2 proportion we may conclude that a small lattice distortion can change the individual $\Delta \varepsilon$ but not the sum $4 \Delta \varepsilon_{\text {api }}+8 \Delta \varepsilon_{\text {equa }}$ which is roughly constant (but only in linear approximation). Thus, $4 \Delta \varepsilon_{\text {api }}+8 \Delta \varepsilon_{\text {equa }}$ (and consequently the total correlation energy as well) is characterized by small linear terms and by large quadratic terms. The physical explanation is given by a competition between the bonds $\mathrm{Ti}$ - apical $\mathrm{O}$ and $\mathrm{Ti}$ - equatorial O. Suppose we distort the crystal in such a way that only the internal coordinate $r_{1}$ increases. The apical oxygens are a little further apart and the interatomic van der Waals-like correlation energy contained within $\Delta \varepsilon_{a p i}$ decreases in magnitude. At the same time, the polarizing influence of the $\mathrm{Ti}$ ions on the charge clouds of the apical oxygen anions decreases; this leads to a charge displacement from these anions towards their other Ti neighbours, with respect to which they are in equatorial positions, and, as a result, the electron correlation contained in $\left|\Delta \varepsilon_{\text {equa }}\right|$ is getting bigger. Analogous effects (of opposite sign) arise with a change of $r_{2}$, with the exception that the two equatorial Ti neighbours of an oxygen anion are moved simultaneously, which leads to a smaller prefactor of $\Delta r_{2}$, in the expression for $\Delta \varepsilon_{\text {equa }}$, as compared to that of $\Delta r_{1}$ for $\Delta \varepsilon_{a p i}$. The two discussed types of correlation-energy change are opposite and in linear approximation almost cancel.

In conclusion we have shown that electron correlations do not change the lattice geometry in a major way but are important for other ground-state properties - the cohesive energy and bulk compressibility which we ob- 
tained are close to the experimental values. In view of these results, we conclude that the application of the incremental scheme to rutile yields new insights (in spite of the fact that we were forced to go to approximations which are problematic from the methodological point of view). In addition we gained valuable experience about how to apply this scheme to mixed covalent-ionic systems with fractional ion charges.

\section{ACKNOWLEDGMENTS}

We would like to thank Dr. M. Dolg, of the MPI-PKS (Dresden), for valuable discussions.

${ }^{1}$ M. D. Towler, N. L. Allan, N. M. Harrison, V. R. Saunders, W. C. Mackrodt, and E. Aprà, Phys. Rev. B 50, 5041 (1994)

${ }^{2}$ K. Doll, M. Dolg, P. Fulde, and H. Stoll, Phys. Rev. B 55, 10282 (1997).

${ }^{3}$ B. Silvi, N. Fourati, R. Nada, and C. R. A. Catlow, J. Phys. Chem. Solids 52, 1005 (1991).

${ }^{4}$ P. Reinhardt, B. A. Heß, and M. Causà, Int. J. Quant. Chem. 58, 297 (1996), see also P. Reinhardt, Ph.D Thesis, University of Bonn (1995) (unpublished).

${ }^{5}$ P. I. Sorantin and K. Schwarz, Inorg. Chem. 31, 567 (1992).

${ }^{6}$ K. M. Glassford and J. R. Chelikowsky, Phys. Rev. B 46, 1284 (1992).

7 J. Muscat, N. M. Harrison, and G. Thornton, in preparation

${ }^{8}$ H. Stoll, Phys. Rev. B 46, 6700 (1992).

${ }^{9}$ H. Stoll, Chem. Phys. Lett. 191548 (1992).

${ }^{10}$ H. Stoll, J. Chem. Phys. 97, 8449 (1992).

${ }^{11}$ B. Paulus, P. Fulde, and H. Stoll, Phys. Rev. B 51, 10572 (1995).

12 B. Paulus, P. Fulde, and H. Stoll, Phys. Rev. B 54, 2556 (1996); S. Kalvoda, B. Paulus, P. Fulde, and H. Stoll, Phys. Rev. B 55, 4027 (1997).

13 B. Paulus, Fa-Jian Shi, and H. Stoll, J. Phys.: Cond. Matter 9, 2745 (1997).

${ }^{14}$ K. Doll, M. Dolg, P. Fulde, and H. Stoll, Phys. Rev. B 52, 4842 (1995).

${ }^{15}$ K. Doll, M. Dolg, and H. Stoll, Phys. Rev. B 54, 13529 (1996).

${ }^{16}$ K. Doll and H. Stoll, Phys. Rev. B 56, 10121 (1997).

${ }^{17}$ M. Albrecht, B. Paulus, and H. Stoll, Phys. Rev. B 56, 7339 (1997).

${ }^{18}$ CRC Handbook of Chemistry and Physics, 77th edition, Editor: D. R. Lide, CRC Press, Boca Raton, New York, London, Tokyo, 1997; The experimental X-ray data obtained for temperatures in range from $30^{\circ} \mathrm{C}$ to $500^{\circ} \mathrm{C}$ were extrapolated to $T=0 \mathrm{~K}$ by assuming a $T^{3}$ dependence of the thermal expansion coefficients. 
19 T. Schork, Ph.D. thesis, University of Stuttgart (1992) (unpublished).

${ }^{20}$ P. Fulde, Electron Correlations in Molecules and Solids, Springer Series in Solid-State Sciences, Vol. 100, Springer, Berlin, Heidelberg, New York, $3^{\text {rd }}$ Edition (1995).

${ }^{21}$ C. Pisani, R. Dovesi, and C. Roetti, Hartree-Fock Ab initio Treatment of Crystalline Systems, Lecture Notes in Chemistry, vol. 48, Edited by G. Berthier et al., Springer, Berlin 1988.

${ }^{22}$ R. Dovesi, V. R. Saunders, C. Roetti, M. Causà, N. M. Harrison, R. Orlando, and E. Aprà, Computer Code CRYSTAL95, Theoretical Chemistry Group - University of Torino, Italy and CCLRC Daresbury Laboratory, UK, 1996.

${ }^{23}$ M. Dolg, U. Wedig, H. Stoll, and H. Preuss, J. Chem. Phys. 86, 866 (1987).

${ }^{24}$ M. Causà, R. Dovesi, C. Pisani, and C. Roetti, Phys. Rev. B 33, 1308 (1986).

${ }^{25}$ We added two $s p$ exponents $(0.21,0.07)$ and one $d$ exponent (0.1) to the titanium basis set, the oxygen basis set was augmented with two additional $s p$ exponents $(0.1,0.04)$. The energies of the neutral atoms in their ground state are $-57.72907 \mathrm{H}(\mathrm{Ti})$ and $-74.79135 \mathrm{H}(\mathrm{O})$.

${ }^{26}$ T. H. Dunning, Jr., J. Chem. Phys. 90, 1007 (1989); R. A. Kendall, T. H. Dunning, Jr., and R. J. Harrison, J. Chem. Phys. 96, 6796 (1992).

${ }^{27}$ R. C. Bartlett, J. Chem. Phys. 93, 1697 (1989); J. Paldus, in "Methods in Computational Physics", Editors: S. Wilson and G. H. F. Diercksen, Plenum Press, New York, 1992.

28 J. Noga and R. J. Bartlett, J. Chem. Phys. 86, 7041 (1987); ibid. 93, 1697 (1989); G. E. Scuseria and H. F. Schaefer III, Chem. Phys. Lett. 152, 382 (1988);K. Raghavachari, G. W. Trucks, J. A. Pople, and M. Head-Gordon, Chem. Phys. Lett. 157, 479 (1989).

${ }^{29}$ MOLPRO is a package of $a b$ initio programs written by H.-J. Werner and P.J. Knowles, with contributions from J. Almlöf, R. D. Amos, M. J. O. Deegan, F. Eckert, S. T. Elbert, C. Hampel, W. Meyer, A. Nicklass, K. Peterson, R. M. Pitzer, A. J. Stone, P. R. Taylor, M. E. Mura, P. Pulay, M. Schuetz, H. Stoll, T. Thorsteinsson, and D. L. Cooper.

${ }^{30}$ C. Hampel, K. Peterson, and H.-J. Werner, Chem. Phys. Lett. 190, 1 (1992); P. J. Knowles, C. Hampel, and H.-J. Werner, J. Chem. Phys. 99, 5219 (1993); M. J. O. Deegan and P. J. Knowles, Chem. Phys. Lett. 227, 321 (1994).

${ }^{31}$ P. J. Hay and R. W. Wadt, J. Chem. Phys. 82, 270 (1985).

${ }^{32}$ Additional calculations (with somewhat reduced basis sets) for $\mathrm{a} \mathrm{TiO}_{6}$ cluster featuring the full crystal coordination of a $\mathrm{Ti}^{4+}$-like entity lead to an even larger reduction of the $\mathrm{TiO}$ increment, by $\sim 5 \mathrm{mH}$, thus still improving the cancellation with the changes in the O-O increments.

${ }^{33}$ I. Jacob, R. Moreh, O. Shahal, and A. Wolf, Phys. Rev. B 35, 8 (1987).

${ }^{34}$ C. E. Moore, Atomic Energy Levels, NBS Circular No. 467, US Government Printing Office, Washinton, DC, 1958; compare also NBS electronic database: http://aeldata.nist.gov 
TABLE I. Ab initio results for rutile

\begin{tabular}{|c|c|c|c|c|c|}
\hline & $\mathrm{SCF}^{\mathrm{a}}$ & $\mathrm{SCF}^{\mathrm{b}}$ & SCF present work & $\mathrm{SCF}+\operatorname{CCSD}(\mathrm{T})$ & experiment \\
\hline$\overline{a_{0}{ }^{\mathrm{c}}}$ & 4.559 & 4.555 & 4.529 & 4.548 & 4.592 \\
\hline$c_{0}{ }^{\mathrm{c}}$ & 3.027 & 3.024 & 3.088 & 2.993 & 2.958 \\
\hline$x_{0}$ & 0.3048 & 0.3061 & 0.3052 & 0.3046 & 0.3048 \\
\hline$B^{\mathrm{d}}$ & 2.81 & 2.79 & 3.04 & $2.36^{\mathrm{f}}$ & 2.39 \\
\hline$B_{I S O}{ }^{\mathrm{d}}$ & 3.09 & 3.08 & 3.15 & 2.45 & - \\
\hline$\Delta E_{B}{ }^{\mathrm{e}}$ & 1.025 & 1.175 & 0.834 & 1.422 & 1.470 \\
\hline
\end{tabular}

${ }^{a}$ Ref. 1 data (all-electron calculations)

${ }^{\mathrm{b}}$ Ref. 1 data (pseudopotentials on $\mathrm{Ti}$ and $\mathrm{O}$ )

c units are $\AA$

d units are Mbar

e $\Delta E_{B}$ is cohesive energy per unit cell; in Hartree units, including zero point vibrations with a Debye approximation and a Debye tempergture of $530 \mathrm{~K} 33$; the energies of the free atoms are averaged over $J$ with the appropriate experimental spin-orbit contributions 34 )

f The estimated error bar for $B$ is \pm 0.06 
TABLE II. Selected correlation-energy increments in rutile, from CCSD calculations (SCF equilibrium geometry from Ref. 4, 2nd column in Table I; atomic units). For the free atoms, we obtain correlation energies of $-0.1714 \mathrm{H} \mathrm{for} \mathrm{O}$, and $-0.4040 \mathrm{H}$ for $\mathrm{Ti}$, at the CCSD level.

\begin{tabular}{|c|c|c|c|}
\hline & cluster & increment & $\begin{array}{l}\text { weight factor } \\
\text { per unit cell }\end{array}$ \\
\hline \multirow[t]{4}{*}{$\overline{\Delta \varepsilon(O)}$} & $\mathrm{O}$ & -0.2613 & 4 \\
\hline & $\mathrm{Ti}-\mathrm{O}^{\mathrm{a}}$ & -0.2692 & \\
\hline & $\mathrm{O}-\mathrm{O}^{\mathrm{c}}$ & -0.2607 & \\
\hline & $\mathrm{O}-\mathrm{Ti}-\mathrm{O}^{\mathrm{d}}$ & -0.2667 & \\
\hline \multirow[t]{6}{*}{$\overline{\Delta \varepsilon(T i)}$} & $\mathrm{Ti}$ & -0.3117 & 2 \\
\hline & $\mathrm{Ti}-\mathrm{O}^{\mathrm{a}}$ & -0.3054 & \\
\hline & $\mathrm{Ti}-\mathrm{O}^{\mathrm{b}}$ & -0.3052 & \\
\hline & $\mathrm{O}-\mathrm{Ti}-\mathrm{O}^{\mathrm{d}}$ & -0.3009 & \\
\hline & $\mathrm{O}-\mathrm{Ti}-\mathrm{O}^{\mathrm{e}}$ & -0.3006 & \\
\hline & $\mathrm{Ti}-\mathrm{O}-\mathrm{Ti}^{\mathrm{f}}$ & -0.3062 & \\
\hline \multirow[t]{4}{*}{$\overline{\Delta \varepsilon\left(T i-O_{\text {equa }}\right)^{\mathrm{b}}}$} & $\mathrm{Ti}-\mathrm{O}^{\mathrm{b}}$ & -0.0239 & 8 \\
\hline & $\mathrm{O}-\mathrm{Ti}-\mathrm{O}^{\mathrm{d}}$ & -0.0206 & \\
\hline & $\mathrm{O}-\mathrm{Ti}-\mathrm{O}^{\mathrm{e}}$ & -0.0204 & \\
\hline & $\mathrm{Ti}-\mathrm{O}-\mathrm{Ti}^{\mathrm{f}}$ & -0.0208 & \\
\hline \multirow[t]{2}{*}{$\overline{\Delta \varepsilon(T i-T i)^{g}}$} & $\mathrm{Ti}-\mathrm{Ti}^{\mathrm{b}}$ & -0.0001 & 2 \\
\hline & Ti-O-Ti ${ }^{f}$ & -0.0001 & \\
\hline \multirow[t]{2}{*}{$\overline{\Delta \varepsilon(O-O)^{\mathrm{c}}}$} & $\mathrm{O}-\mathrm{O}^{\mathrm{c}}$ & -0.0051 & 2 \\
\hline & $\mathrm{O}-\mathrm{Ti}-\mathrm{O}^{\mathrm{e}}$ & -0.0093 & \\
\hline \multirow[t]{2}{*}{$\overline{\Delta \varepsilon(O-O)^{\mathrm{h}}}$} & $\mathrm{O}-\mathrm{O}^{\mathrm{h}}$ & -0.0029 & 16 \\
\hline & $\mathrm{O}-\mathrm{Ti}-\mathrm{O}^{\mathrm{d}}$ & -0.0066 & \\
\hline$\overline{\Delta \varepsilon(O-T i-O)^{\mathrm{d}}}$ & $\mathrm{O}-\mathrm{Ti}-\mathrm{O}^{\mathrm{d}}$ & +0.0012 & 16 \\
\hline \multicolumn{4}{|c|}{ b $\mathrm{Ti}-$ nearest neighbour equatorial oxygen (distance -3.70 a.u.) } \\
\hline \multicolumn{4}{|c|}{ f $\mathrm{Ti}-\mathrm{O}-\mathrm{Ti}$ (distances: 3.72 and 3.70 a.u.) } \\
\hline \multicolumn{4}{|c|}{ g nearest neighbour Ti-Ti pair (distance - 5.71 a.u.) } \\
\hline h next nearest neig & in (diat & & \\
\hline
\end{tabular}

FIG. 1. The rutile structure with oxygen (open circles) and titanium atoms (filled circles). 


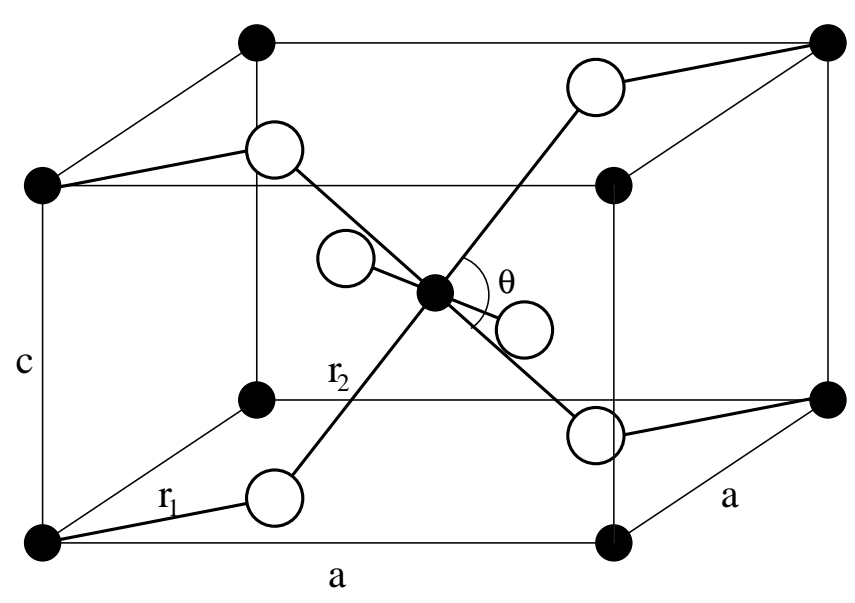

